\title{
Role of biofilm in Staphylococcus aureus and Staphylococcus epidermidis ventricular assist device driveline infections
}

\author{
Faustino A. Toba, PhD, ${ }^{\mathrm{a}}$ Hirokazu Akashi, MD, ${ }^{\mathrm{b}}$ Carlos Arrecubieta, $\mathrm{PhD},{ }^{\mathrm{a}}$ and Franklin D. Lowy, $\mathrm{MD}^{\mathrm{a}, \mathrm{c}}$
}

Objective: Infections, especially those involving drivelines, are among the most serious complications that follow ventricular assist device implantation. Staphylococci are the most common causes of these infections. Once driveline infections are established, they can remain localized or progress as an ascending infection to cause metastatic seeding of other tissue sites. Although elaboration of biofilm appears to be critical in prosthetic device infections, its role as a facilitator of staphylococcal infection and migration along the driveline and other prosthetic devices is unclear.

\begin{abstract}
Methods: A murine model of driveline infection was used to investigate staphylococcal migration along the driveline. A biofilm-producing strain of Staphylococcus epidermidis and a Staphylococcus aureus strain and its intercellular adhesion gene cluster (ica)-negative (biofilm-deficient) isogenic mutant were used in these studies. Bacterial density on the driveline and the underlying tissue was measured over time. Scanning electron microscopy was used to examine the morphology of $S$ epidermidis biofilm formation as the infection progressed.

Results: The biofilm-deficient $S$ aureus mutant was less effective at infecting and migrating along the driveline than the wild-type strain over time. However, the ica mutation had no effect on the ability of the strain to infect underlying tissue. $S$ aureus exhibited more rapid migration than $S$ epidermidis. Scanning electron microscopy revealed the deposition of host matrix on the Dacron material after implantation. This was followed by elaboration of a bacterial biofilm that correlated with more rapid migration along the driveline.
\end{abstract}

Conclusions: Biofilm formation is a critical virulence determinant that facilitates the progression of drivelines infections. (J Thorac Cardiovasc Surg 2011;141:1259-64)

Ventricular assist devices (VADs) are used to improve cardiac function in patients with end-stage congestive heart failure. ${ }^{1-3}$ Originally designed as a bridge to transplantation, the use of VADs as destination therapy (for patients who are not transplant candidates or who have refractory heart failure) is increasing. ${ }^{1,2}$ Infections are among the most common and serious complications in patients $(10 \%-40 \%)$ after VAD implantation. ${ }^{4}$ The presence of these infections might delay transplantation in bridge-to-transplantation patients and is a major cause of death in destination patients. Despite a reduction in the overall number of VAD-related infections, the driveline remains a common site of infection $(21 \%$, as reported by the INTERMACS Registry at www.intermacs. org). ${ }^{3}$ The driveline exit site is accessible to commensal flora and susceptible to trauma that can interfere with tissue integration of the driveline. ${ }^{5}$ The ability of bacteria to cause

\footnotetext{
From the Division of Infectious Diseases, Department of Medicine, ${ }^{\mathrm{a}}$ and the Department of Pathology, ${ }^{\mathrm{c}}$ College of Physicians and Surgeons, Columbia University, New York, NY; and the Department of Surgery, ${ }^{\mathrm{b}}$ New York Presbyterian Hospital, Columbia University, New York, NY.

Disclosures: Authors have nothing to disclose with regard to commercial support.

Dr Arrecubieta is currently affiliated with Regeneron Pharmaceuticals, Inc, Tarrytown, NY.

Received for publication March 24, 2010; revisions received June 22, 2010; accepted for publication July 4, 2010; available ahead of print Aug 16, 2010.

Address for reprints: Franklin D. Lowy, MD, Department of Medicine, Columbia University, 630 W 168th St, New York, NY 10032 (E-mail: fl189@columbia.edu). $0022-5223 / \$ 36.00$

Copyright (c) 2011 by The American Association for Thoracic Surgery doi:10.1016/j.jtcvs.2010.07.016
}

these infections is due in part to their ability to adhere to and colonize both skin and device surfaces by using the transcutaneous driveline of VADs as a portal of entry. 6,7

The predominant pathogens associated with device infections are biofilm-forming Staphylococcus epidermidis and Staphylococcus aureus; both are part of the host commensal flora. ${ }^{8}$ Infection results from the ability of these bacteria to initially colonize the device surface and then migrate along its surface into the underlying host tissue. ${ }^{6,9,10}$ Biofilms appear to contribute to this process. ${ }^{5,11,12}$ They constitute a consortium of surface-attached bacteria encased in a selfproduced extracellular polymeric substance. ${ }^{5,12-14}$ When a VAD is implanted, a series of host molecules, such as fibronectin, fibrin, collagen, lipids, and inorganic ions, are deposited on the different VAD surfaces, creating a host tissue matrix. ${ }^{5,7,12-14}$ This matrix facilitates the attachment of biofilm-forming bacteria.

In staphylococci, the intercellular adhesion gene cluster (ica) comprises 4 genes (icaADBC) that direct the production of a polysaccharide that mediates intercellular adhesion and is regarded as one of the essential factors for biofilm formation. ${ }^{15}$

Although most driveline infections remain localized to the entry site, they can also spread to other tissue sites. One hypothesis for this process is that there is bacterial biofilm expansion that enables bacteria to migrate along the driveline. With this in mind, we investigated the contribution $S$ aureus and $S$ epidermidis biofilm to the initiation and progression of driveline infections using an in vivo model of driveline infection. 


\section{Abbreviations and Acronyms \\ $\mathrm{CFU}=$ colony-forming units \\ ica $=$ intercellular adhesion gene cluster \\ $\mathrm{VAD}=$ ventricular assist device}

\section{MATERIALS AND METHODS}

\section{Bacterial strains and growth conditions}

$S$ epidermidis strain 9491 (SE9491) ${ }^{16}$ and $S$ aureus isogenic strains RN450 (SA450) and RN450 ( $\Delta i c a$; SA $\Delta$ ica; kindly provided by Chia Y. Lee) ${ }^{17,18}$ were grown in tryptic soy broth (supplemented with glucose $0.25 \%$ for SE9491) at $37^{\circ} \mathrm{C}$ with aeration (Table 1). The presence of ica in SA450 and SE9491 was confirmed by means of polymerase chain reaction by using the following primers: $i c a$ forward primer, $5^{\prime}$-GAT TGT ATT AGC TGT AGC TAC- ${ }^{\prime} 3$, and ica reverse primer, 5'-CAA CTG CAG CTG ATT TCG CCC ACC GCG TG-'3. For plate counts, mannitol salt agar plates or tryptic soy agar plates were incubated overnight at $37^{\circ} \mathrm{C}$. A growth curve (tryptic soy broth at $37^{\circ} \mathrm{C}$ ) was performed to assess the effect of the ica deletion on SA $\Delta$ ica. No difference was found in the rate of growth between the isogenic pair.

\section{In Vivo Infection Model}

The murine model of driveline infection has been previously described. ${ }^{7,19}$ Briefly, 15-mm-long Dacron (kindly provided by Thoratec, Pleasanton, Calif)-coated drivelines were implanted transcutaneously in the backs of C57BL/6J mice. The model is shown in Figure 1. The internal (subcutaneous) part of the drivelines was $10 \mathrm{~mm}$ in length. The day of implantation was considered day -2 . Two days after implantation (day 0 ), the skin surrounding the exposed driveline was inoculated with a bacterial suspension $(50 \mu \mathrm{L})$ containing $5 \times 10^{7}$ colony-forming units (CFU) of SE9491 or $5 \times 10^{6} \mathrm{CFU}$ of $S$ aureus (suspended in phosphate-buffered saline). No perioperative antibiotics were administered to the mice. Two days after bacterial challenge (day 2), the drivelines were explanted, the external portion was sterilely separated, and the internal portions were processed (see below). These studies were reviewed and approved by the Columbia University Institutional Animal Care and Use Committee.

The drivelines were washed twice with phosphate-buffered saline $(1 \mathrm{~mL})$, and then adherent bacteria were harvested by vortexing in $0.05 \%$ trypsin-ethylenediamine tetraacetic acid $(0.5 \mathrm{~mL})$. Dilutions of the samples were plated onto mannitol salt agar plates, and bacterial cell counts were determined. The tissue underlying the driveline was also collected from each mouse and weighed. The homogenized samples were weighed and then plated onto mannitol salt agar plates; bacterial counts were determined and then normalized by sample weight. Two independent experiments were performed with a total of 20 mice per strain.

For the bacterial migration studies, the driveline size was increased to 20 $\mathrm{mm}$ in length, increasing the subcutaneous portion to $15 \mathrm{~mm}$ in length. To measure bacterial migration along the driveline over time, we divided the driveline into three 5-mm segments. The 3 subcutaneous sections were defined as proximal, medial, and distal based on their position with respect to the transcutaneous entry point. Infection of the driveline was carried out as outlined above. The bacterial inoculum was increased to $1 \times 10^{7} \mathrm{CFU}$. Drivelines were explanted at days 0, 2, 6, 9, and 12. Each section (proximal, medial, and distal) was processed separately. Results were expressed as a ratio by comparing the bacterial counts of each section to the total number of bacteria found per driveline (proximal + medial + distal).

Tissue samples of kidney, liver, and spleen were collected and processed in a similar manner as the underlying muscle tissue described above to assess for possible metastatic seeding.

\section{Scanning Electron Microscopy}

Sections of explanted drivelines were fixed with glutaraldehyde $(2 \%)$ in cacodylate buffer, as previously described. ${ }^{20}$ A JEOL JSM6400 Scanning Electron Microscope (Peabody, Mass) was used. Three micrographs were obtained from each section of 2 different drivelines. Images of control driveline samples (nonimplanted and implanted but noninfected) were compared with images obtained from infected drivelines at different time points (days 6 and 12 after infection).

\section{Statistical Analysis}

Data were analyzed by using an unpaired Student $t$ test.

\section{RESULTS}

\section{Role of Biofilm in Driveline Infections}

The role that biofilm contributes to bacterial density on the drivelines and in the surrounding tissue was assessed by using both the isogenic strain of $S$ aureus and a biofilm-positive $S$ epidermidis strain. The average bacterial counts on the explanted drivelines for the biofilm-forming strain SA450 was $5 \times 10^{4} \mathrm{CFU}$ per driveline; this was significantly higher than those for the biofilm-negative strain $\mathrm{SA} \Delta$ ica $\left(2 \times 10^{4}\right.$ per driveline, Figure $\left.2, P<.05\right)$. Despite the difference in adherence to the driveline, the presence of bacteria in the underlying tissue was similar for the isogenic strains $(P>.05$, Figure 2). Although biofilm positive, SE9491 had fewer bacterial counts than SA450 on both the drivelines ( $3 \times 10^{4}$ per driveline) and in the underlying tissue $\left(1 \times 10^{4} \mathrm{CFU} / \mathrm{g}, P<.05\right.$, Figure 2$)$. This was despite the use of a higher initial bacterial inoculum.

\section{Role of Biofilm as a Facilitator of Migration Along the Driveline}

Bacterial migration along the driveline was studied over time (Figure 3). With SA450, total bacterial density went from $2.6 \times 10^{4} \mathrm{CFU}$ per driveline at day 2 to $4.8 \times 10^{5}$ CFU per driveline by day 12 , almost doubling every 2 days (Figure 4, A). In contrast, the bacterial density for

TABLE 1. Description of bacterial strains used in this study

\begin{tabular}{llll}
\hline \multicolumn{1}{c}{ Species } & \multicolumn{1}{c}{ Strain } & \multicolumn{1}{c}{ Phenotype } & Reference \\
\hline Staphylococcus aureus & RN450 & Biofilm-forming wild type & Chia Y. Lee, University of Arkansas \\
& SA450 & & \\
& RN450 $(\Delta \mathrm{ica})$ & Biofilm-deficient strain & Chia Y. Lee, University of Arkansas \\
& SA $\Delta$ ica & & McCrea, 2000 \\
& 9491 & Biofilm-positive laboratory strain & \\
\hline
\end{tabular}




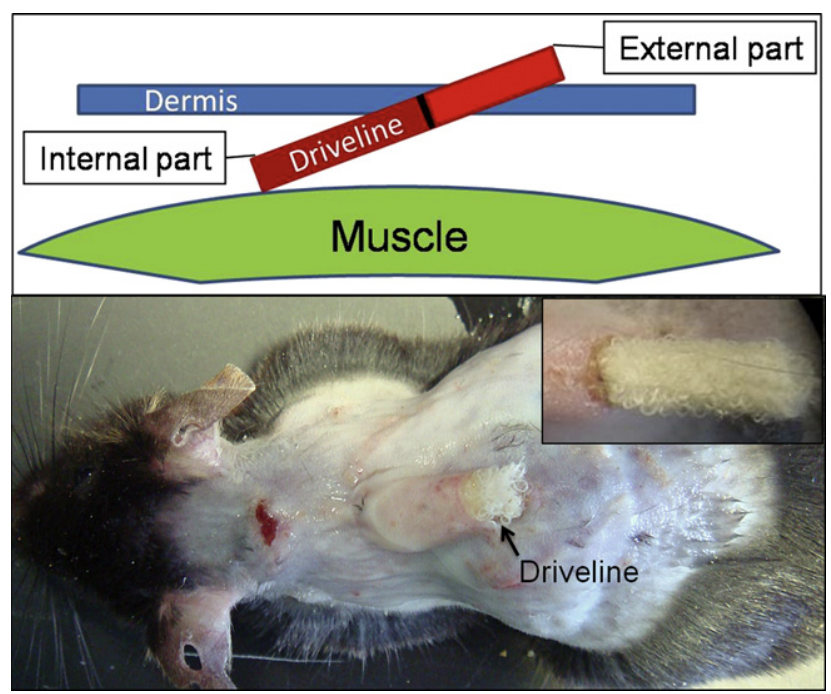

FIGURE 1. Description of the driveline model. The upper figure displays a schematic representation of placement of the driveline into the back of the mouse. The lower figure displays the driveline implanted into a mouse. The inset provides a more detailed view of the implanted driveline. Reproduced with the permission of the Journal of Heart and Lung Transplantation, Elsevier.

SA $\Delta$ ica, although increasing throughout the assay from $9 \times 10^{3} \mathrm{CFU}$ per driveline at day 2 to $5.4 \times 10^{4} \mathrm{CFU}$ per driveline at day 12, was 10-fold lower than that of SA450 (Figure 4, B).

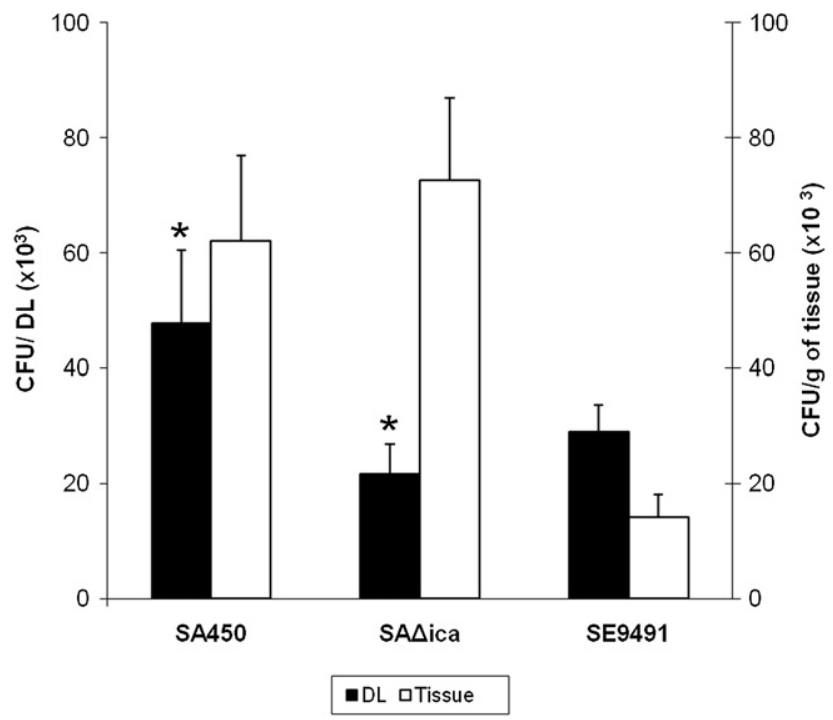

FIGURE 2. Bacterial density on drivelines and in tissue harvested from mice infected with $S$ aureus or $S$ epidermidis. Mice with implanted drivelines were infected with SA450 $(n=20)$ or SA $\Delta$ ica $(n=20)$. The drivelines and samples of the underlying muscle were collected at 48 hours. Cells were enumerated by means of plate counting. There was a statistically higher number of bacteria adherent to the SA450-infected driveline than with SA $\Delta$ ica $(P<.05)$. There was no difference in bacterial density in the underlying tissue. Bacterial density was lower in both the driveline and tissue for $S$ epidermidis strain SE9491. CFU, Colony-forming units; $D L$, driveline.
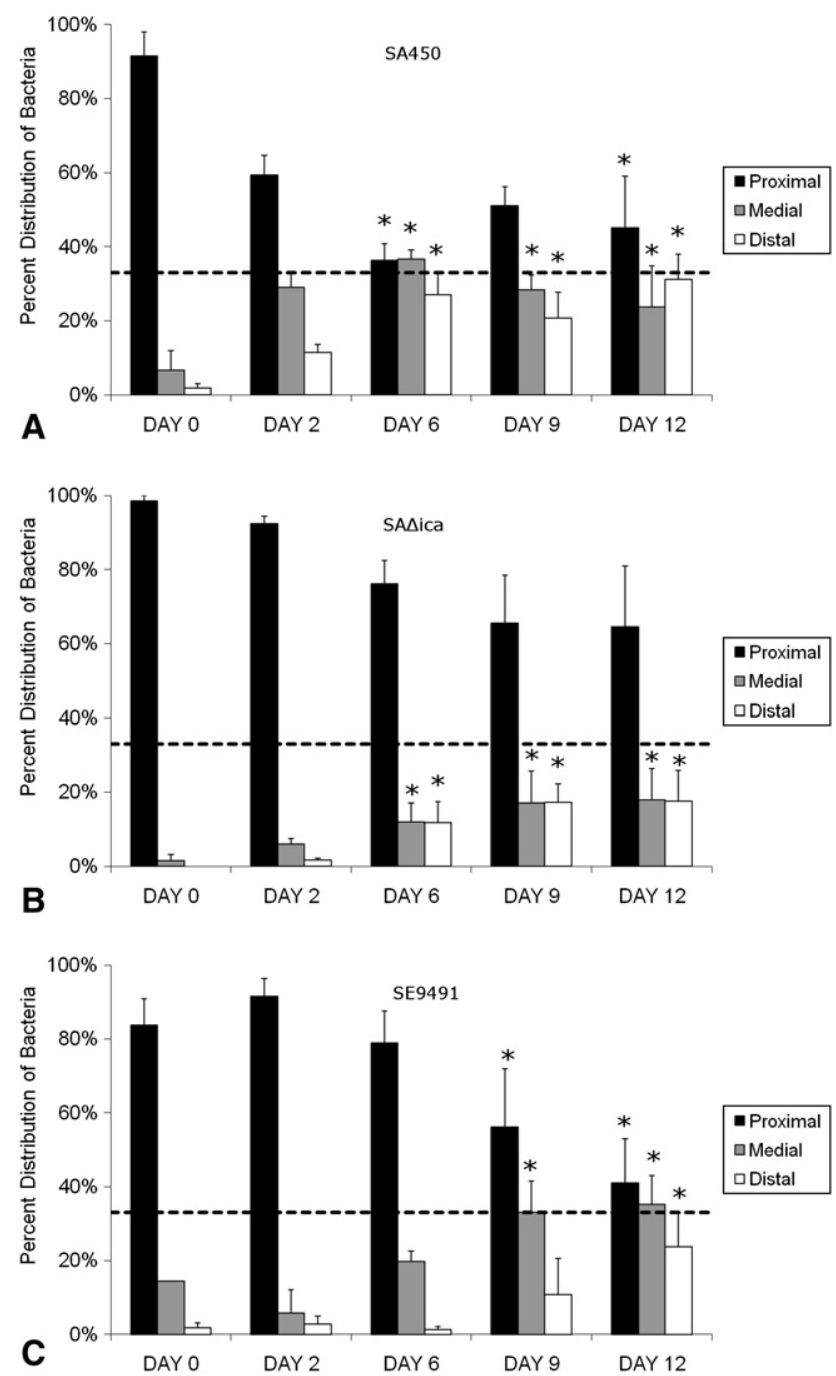

FIGURE 3. The role of biofilm in bacterial migration along the driveline Mice were implanted with 20-mm drivelines and exposed to SA450 (A), SA $\Delta$ ica (B), or SE9491 (C). Drivelines were harvested at different time points after implantation, and the number of bacteria adherent to different segments of the driveline (proximal, medial, and distal) was determined. A ratio of adherence was determined (number of bacteria adherent to the driveline segment/total number bacteria on the driveline (proximal + medial + distal). The experiments were done in duplicate with 6 mice per treatment. Similar ratios are noted with an asterisk $(P>.05)$. The horizontal line indicates what would represent an even distribution of adherent bacteria per section. When the distribution of the bacteria along the catheter of the isogenic pair is compared, SA450 (A) versus SA $\Delta$ ica (B), there is a significantly higher number of bacteria found distally in the wild-type SA450 $(P<.05)$.

At an early stage of infection (day 2), $60 \%$ of SA450 was located on the proximal section, $30 \%$ on the medial section, and $10 \%$ on the distal section. By day 6 , SA450 was uniformly distributed among the 3 driveline sections $(P>$ .05 ; Figure $3, A)$. Although SA $\Delta$ ica was also found in all 3 sections (Figure 3, B), this biofilm-deficient mutant never 

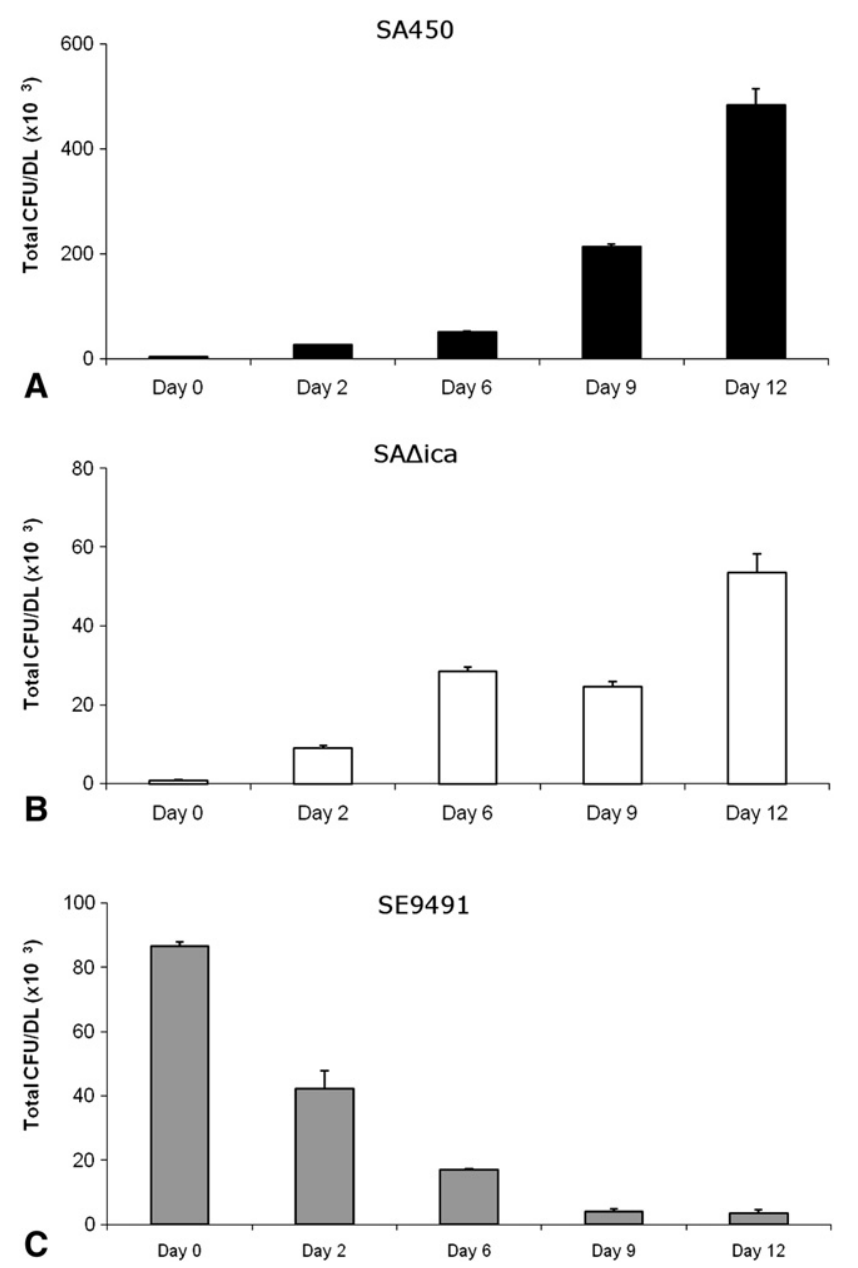

FIGURE 4. Bacterial density on drivelines infected with $S$ aureus or S epidermidis over time. Mice were implanted with 20-mm drivelines and exposed to SA450 (A), biofilm-deficient SADica (B), and SE9491 (C). At different time points, drivelines were explanted, and the number of bacteria adherent to the 3 sections (proximal, medial, and distal) was counted. The results display the total number of bacteria found on each driveline. The total number of bacteria increased in both $S$ aureus strains, whereas there was a decrease in the number of $S$ epidermidis strain SE9491 on the infected drivelines, suggesting diminished virulence for the latter. $C F U$, Colonyforming units; $D L$, driveline.

achieved an even distribution along the drivelines $(P<.05)$. Even by day $12,64 \%$ of the CFU per driveline of SA $\Delta$ ica was still limited to the proximal section of the CFU per driveline (Figure 3, $B$ ).

The biofilm-forming strain SE9491 was also evaluated in the in vivo driveline assay. In contrast to $S$ aureus, the total bacterial counts decreased over the 12-day study, suggesting a more indolent infection. The bacterial density of SE9491 started at $8.6 \times 10^{4} \mathrm{CFU}$ per driveline (day 2) and decreased to $3.8 \times 10^{3} \mathrm{CFU}$ per driveline by days 9 and 12 , nearly 100 -fold lower than SA450 bacterial density at the end of the experiment. Despite this, a similar pattern to that of
SA450 was observed regarding bacterial distribution. By day 12 , bacteria were evenly distributed in all 3 driveline sections (Figure 3, $C ; P>.05$ ).

No bacteria were found in samples taken from the kidney, liver, or spleen.

\section{Scanning Electron Microscopy}

Scanning electron microscopy was used to visualize and characterize the progression of the driveline infection over time. The Dacron fibers examined before implantation appeared smooth and uniform in size (Figure 5). Electron micrographs of implanted uninfected drivelines taken at day 6 revealed extracellular matrix components and host cells coating the Dacron fibers (Figure 5). Micrographs of SE9491-infected drivelines at day 6 showed formation of biofilm in the proximal and medial sections. The distal sections of these drivelines were almost free of SE9491 and showed no biofilm structures at day 6 (Figure 5). By day 12 , uninfected drivelines were covered with an extensive extracellular matrix populated with adherent cells distributed throughout the implanted material, whereas infected drivelines showed a more developed biofilm structure in both the proximal and medial sections than at day 6 (Figure 5). The bacterial biofilm was far more extensively developed, with an increased number of bacteria visible within its structure. In addition, by day 12 , the biofilm extended to the distal section of the infected drivelines (Figure 5).

\section{DISCUSSION}

Driveline infections continue to be the single most common infectious complication of VAD implantation. Fifteen percent to $20 \%$ of patients have these infections, and in contrast with other VAD-related infections, they occur over the entire lifetime of the VAD. ${ }^{3}$ There is limited understanding of the pathogenesis of these infections. ${ }^{4,7}$

In this study an in vivo murine model was used to examine the role of biofilm formation in the pathogenesis of driveline infections and in particular the role of biofilm in bacterial migration along prosthetic devices. Infection was initiated in this model 48 hours after implantation of the driveline. This time frame was selected because staphylococcal infections in particular often occur shortly after surgical intervention at a time when the internal portions of the device remain accessible to skin commensal flora.

For staphylococci, the ica gene cluster is essential for biofilm formation. ${ }^{17,21,22}$ The ica cluster contains 4 genes (icaADBC) that are responsible for the synthesis of poly$\mathrm{N}$-acetyl glucosamine, the molecule that mediates intercellular adhesion, facilitating biofilm formation. Biofilm provides a potential sanctuary for bacteria, protecting against innate host defense mechanisms, such as complement, white blood cells, and immunoglobulin, as well as reducing the efficacy of antibiotics. ${ }^{8,11,12,14,15}$ Less well established is whether biofilm also facilitates the migration of bacteria along 

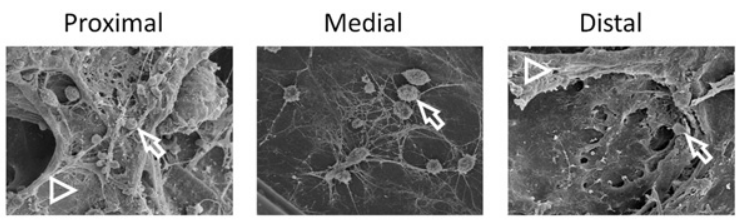

Day 6

(1000x) Uninfected
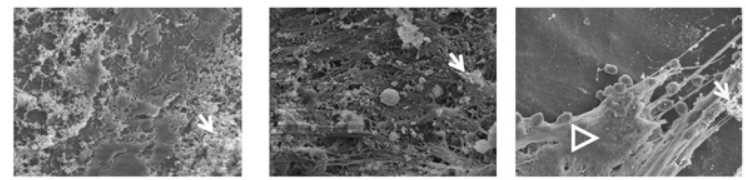

Day 6

(1200x) S. epidermidis
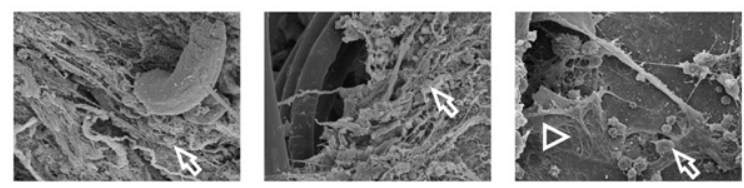

Day 12

(1000x) Uninfected
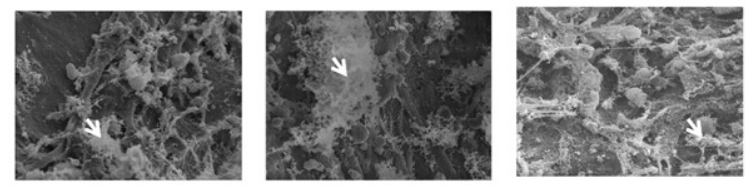

Day 12

(1200x) S. epidermidis

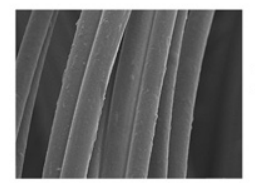

Non-implanted (1000x)

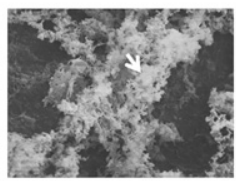

Day 12

(7500x) S. epidermidis

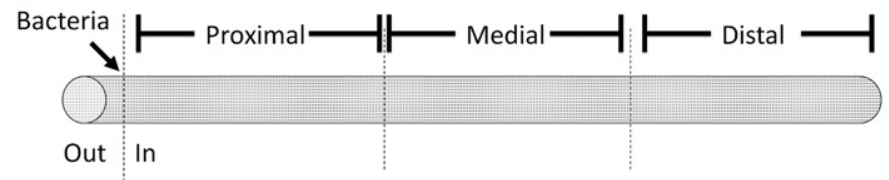

Driveline

FIGURE 5. Scanning electron microscopy of drivelines (proximal, medial, and distal) explanted at different time points after infection with $S$ epidermidis strain SE9491. Dacron fibers from nonimplanted material revealed a smooth surface (bottom left). By day 6, images of the uninfected implanted driveline showed the Dacron coated with matrix material (arrowhead) and host cells (outlined arrows). The SE9491-infected drivelines demonstrate bacteria organized in a biofilm (full arrows) in the proximal and medial sections, whereas the distal sections were mostly free of bacteria. By day 12, uninfected drivelines were covered with a dense tissue matrix with adherent cells (outlined arrows). The infected driveline showed extensive biofilm formation extending to the distal section (full arrows). The images demonstrate that there is a progression that is characterized by the formation of a scaffold of matrix molecules and cells that is first formed along the driveline followed by the adherence of bacteria and elaboration of a biofilm that allows bacterial migration along the driveline.

catheters or, in this instance drivelines, enabling infection to progress to deeper tissue structures or to spread to other organs. This question was addressed in the present study.

The study found that both a biofilm-producing SA450 and a biofilm-positive SE9491 strain migrated along the Dacroncoated driveline and achieved an even distribution along the driveline. The biofilm-deficient strain SA $\Delta$ ica was less capable of infecting the implanted drivelines and remained unevenly distributed. The biofilm-positive SE9491 strain, although establishing a higher initial count on the driveline (a higher initial bacterial inoculum was used), had a lower bacterial density on the drivelines and, in contrast with
S aureus, lost density over time. This suggests that the strain had diminished virulence when compared with $S$ aureus. Overall, the results suggest that ica-dependent biofilm formation played an important role in bacterial migration along the driveline. Interestingly, biofilm formation appeared to have no effect on the ability of the strains to infect the underlying tissue, illustrating the selective virulence of biofilm for prosthetic device infections.

Scanning electron microscopy demonstrated the orderly progression of events leading to infection. Bacteria contaminating the skin migrate across the transcutaneous driveline site, adhere to the host matrix material coating the Dacron, 
replicate, and elaborate a biofilm, allowing the bacteria to both colonize the driveline surface and, as the biofilm expands, to migrate along the driveline. The biofilm architecture was similar to that observed on infected catheters. ${ }^{23,24}$ Control images of uninfected drivelines showed the matrix coating of drivelines after implantation. Host cells associated with the driveline surface deposit fibers and other host molecules that permit integration of the implant into the host tissue. These same molecules (eg, fibronectin and fibrinogen) are also recognized by bacteria as sites for attachment. $^{12,16,25}$

Although this study shows the role of biofilm in driveline infection, it is limited by the use of a single isogenic pair of $S$ aureus strains and a biofilm-producing strain of S epidermidis without an isogenic ica mutant. Creating a biofilmnegative S epidermidis mutant has proved technically difficult because of the limited susceptibility of these strains to transduction and electroporation. Although spread of infection was investigated by using the driveline model, metastatic seeding to other organs (ie, spleen, kidney, and liver) was not detected. The subcutaneous site of driveline implantation in this driveline model made the likelihood of seeding other tissue sites unlikely.

The present study suggests that biofilm, in addition to providing a sanctuary that protects bacteria from host defenses, also facilitates migration of bacteria along prosthetic devices. Access to the underlying tissue enhances the likelihood of a local infection, as well as the potential for spread to other tissue sites. Further studies will need to address host-tissue interactions that facilitate integration of prosthetic materials, such as Dacron, into host tissues and reduce access of bacteria to the underlying tissues. Understanding particular properties of a pathogen that leads to infection is critical to the design of devices that are less susceptible to infection. These determinants can then potentially be used as targets for either preventative or therapeutic interventions.

New therapies that address driveline design, incorporate antimicrobials into prosthetic materials, or reduce biofilm formation might help reduce the frequency of these infections.

\section{References}

1. Park SJ, Tector A, Piccioni W, Raines E, Gelijns A, Moskowitz A, et al. Left ventricular assist devices as destination therapy: a new look at survival. J Thorac Cardiovasc Surg. 2005;129:9-17.

2. Rose EA, Gelijns AC, Moskowitz AJ, Heitjan DF, Stevenson LW, Dembitsky W, et al. Long-term mechanical left ventricular assistance for end-stage heart failure. N Engl J Med. 2001;345:1435-43.
3. Miller LW, Pagani FD, Russell SD, John R, Boyle AJ, Aaronson KD, et al. Use of a Continuous-Flow Device in Patients Awaiting Heart Transplantation. N Engl J Med. 2007;357:885-96.

4. Gordon RJ, Quagliarello B, Lowy FD. Ventricular assist device-related infections. Lancet Infect Dis. 2006;6:426-37.

5. Padera RF. Infection in ventricular assist devices: the role of biofilm. Cardiovasc Pathol. 2006; 15:264-70.

6. Shoham S, Miller LW. Cardiac assist device infections. Curr Infect Dis Rep. 2009;11:268-73.

7. Arrecubieta C, Toba FA, von Bayern M, Akashi H, Deng MC, Naka Y, et al. SdrF, a Staphylococcus epidermidis surface protein, contributes to the initiation of ventricular assist device driveline-related infections. PLoS Pathog. 2009;5:e1000411.

8. O'Gara JP, Humphreys H. Staphylococcus epidermidis biofilms: importance and implications. J Med Microbiol. 2001;50:582-7.

9. Simon D, Fischer S, Grossman A, Downer C, Hota B, Heroux A, et al. Left ventricular assist devices related infection: treatment and outcome. Clin Infect Dis. 2005;40:1108-15.

10. Vilchez RA, McEllistrem MC, Harrison LH, McCurry KR, Kormos RL, Kusne S. Relapsing bacteremia in patients with ventricular assist device: an emergent complication of extended circulatory support. Ann Thorac Surg. 2001;72:96-101.

11. Dasgupta M. Biofilms and infection in dialysis patients. Semin Dial. 2002;15: 338-46.

12. Otto M. Staphylococcus epidermidis - the "accidental" pathogen. Nat Rev Micro. 2009;7:555-67.

13. Costerton JW, Lewandowski Z, Caldwell DE, Korber DR, Lappin-Scott HM. Microbial biofilms. Ann Rev Microbiol. 1995;49:711-45

14. Hall-Stoodley L, Costerton JW, Stoodley P. Bacterial biofilms: from the natural environment to infectious diseases. Nat Rev Micro. 2004;2:95-108.

15. Eftekhar F, Speert DP. Biofilm formation by persistent and non-persistent isolates of Staphylococcus epidermidis from a neonatal intensive care unit. J Hosp Infect. 2009;71:112-6.

16. McCrea KW, Hartford O, Davis S, Eidhin DN, Lina G, Speziale P, et al. The serine-aspartate repeat (Sdr) protein family in Staphylococcus epidermidis. Microbiology. 2000;146:1535-46.

17. Cramton SE, Gerke C, Schnell NF, Nichols WW, Gotz F. The intercellular adhesion (ica) locus is present in Staphylococcus aureus and is required for biofilm formation. Infect Immun. 1999;67:5427-33.

18. Luong TT, Lee CY. Improved single-copy integration vectors for Staphylococcus aureus. J Microbiol Methods. 2007;70:186-90.

19. von Bayern MP, Arrecubieta C, Oz S, Akashi H, Cedeiras M, Naka Y, et al. Development of a murine ventricular assist device transcutaneous drive-line model. J Heart Lung Transplant. 2008;27:812-4.

20. Arrecubieta C, Matsunaga I, Asai T, Naka Y, Deng Mario C, Lowy Franklin D. Vaccination with clumping factor a and fibronectin binding protein A to prevent Staphylococcus aureus infection of an aortic patch in mice. J Infect Dis. 2008;198: 571-5.

21. Resch A, Rosenstein R, Nerz C, Gotz F. Differential gene expression profiling of Staphylococcus aureus cultivated under biofilm and planktonic conditions. Appl Environ Microbiol. 2005;71:2663-76.

22. Ziebuhr W, Heilmann C, Gotz F, Meyer P, Wilms K, Straube E, et al. Detection of the intercellular adhesion gene cluster (ica) and phase variation in Staphylococcus epidermidis blood culture strains and mucosal isolates. Infect Immun. 1997;65: 890-6.

23. Curtin JJ, Donlan RM. Using bacteriophages to reduce formation of catheterassociated biofilms by Staphylococcus epidermidis. Antimicrob Agents Chemother. 2006;50:1268-75.

24. Lorenz U, Hüttinger C, Schäfer T, Ziebuhr W, Thiede A, Hacker J, et al. The alternative sigma factor sigma B of Staphylococcus aureus modulates virulence in experimental central venous catheter-related infections. Microbes Infect. 2008; 10:217-23.

25. O'Connell DP, Nanavaty T, McDevitt D, Gurusiddappa S, Höök M, Foster TJ. The fibrinogen-binding MSCRAMM (clumping factor) of Staphylococcus aureus has a $\mathrm{Ca}^{2+}$-dependent inhibitory site. J Biol Chem. 1998;273:6821-9. 